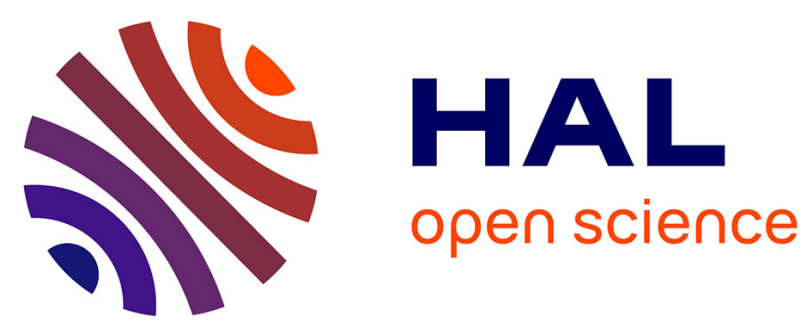

\title{
Acoustic Vibrations of Au Nano-Bipyramids and their Modification under Ag Deposition: a Perspective for the Development of Nanobalances
}

Benoît Dacosta Fernandes, Miguel Spuch-Calvar, Hatim Baida, Mona

Tréguer-Delapierre, Jean Oberlé, Pierre Langot, Julien Burgin

\section{To cite this version:}

Benoît Dacosta Fernandes, Miguel Spuch-Calvar, Hatim Baida, Mona Tréguer-Delapierre, Jean Oberlé, et al.. Acoustic Vibrations of Au Nano-Bipyramids and their Modification under Ag Deposition: a Perspective for the Development of Nanobalances. ACS Nano, 2013, 7 (9), pp.7630-7639. $10.1021 / \mathrm{nn} 402076 \mathrm{~m}$. hal-00874407

\section{HAL Id: hal-00874407 https://hal.science/hal-00874407}

Submitted on 1 Mar 2018

HAL is a multi-disciplinary open access archive for the deposit and dissemination of scientific research documents, whether they are published or not. The documents may come from teaching and research institutions in France or abroad, or from public or private research centers.
L'archive ouverte pluridisciplinaire HAL, est destinée au dépôt et à la diffusion de documents scientifiques de niveau recherche, publiés ou non, émanant des établissements d'enseignement et de recherche français ou étrangers, des laboratoires publics ou privés.

\section{(ㄷ)(1) $\$$}

Distributed under a Creative Commons Attribution - NonCommerciall 4.0 International 


\title{
Acoustic Vibrations of Au Nano- Bipyramids and their Modification under Ag Deposition: a Perspective for the Development of Nanobalances
}

\author{
Benoît Dacosta Fernandes, ${ }^{\dagger}$ Miguel Spuch-Calvar, ${ }^{\ddagger}$ Hatim Baida, ${ }^{\dagger}$ Mona Tréguer-Delapierre, ${ }^{\ddagger}$ Jean Oberlé, ${ }^{\dagger}$ \\ Pierre Langot, ${ }^{\dagger}$ and Julien Burgin ${ }^{\dagger, *}$
}

${ }^{\dagger}$ Univ. Bordeaux, LOMA, UMR 5798, F-33400 Talence, France and ${ }^{\ddagger}$ CNRS, Univ. Bordeaux, ICMCB, UPR 9048, F-33600 Pessac, France

\begin{abstract}
We investigated the acoustic vibrations of gold nanobipyramids and bimetallic gold silver core shell bipyramids, synthesized by wet chemistry techniques, using a high-sensitivity pump probe femtosecond setup. Three modes were observed and characterized in the gold core particles for lengths varying from 49 to $170 \mathrm{~nm}$ and diameters varying from 20 to $40 \mathrm{~nm}$. The two strongest modes have been associated with the fundamental extensional and its first harmonic, and
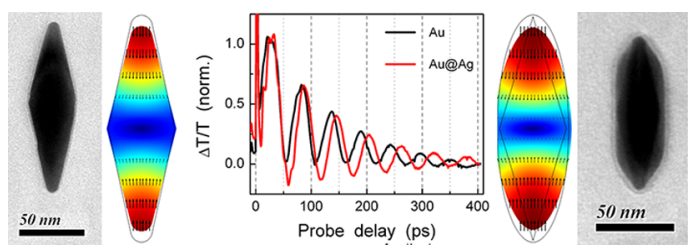

a weak mode has been associated with the fundamental radial mode, in very good agreement with numerical simulations. We then derived linear laws linking the periods to the dimensions both experimentally and numerically. To go further, we investigated the evolution of these modes under silver deposition on gold core bipyramids. We studied the evolution of the periods of the extensional modes, which were found to be in good qualitative agreement with numerical simulations. Moreover, we observed a strong enhancement of the radial mode amplitude when silver is deposited: we are typically sensitive to the deposition of 40 attograms of silver per gold core particle. This opens up possible applications in the field of mass sensing, where metallic nanobalances have an important role to play, taking advantage of their robustness and versatility.
\end{abstract}

KEYWORDS: gold nanoparticles · core shell nanoparticles · time resolved spectroscopy · acoustic vibrations · nanosensors

Acoustic vibrations of nanometric sys-tems have been extensively studied in the recent years, focusing on semiconducting, ${ }^{1-3}$ metallic, $^{4-19}$ and hybrid nanoparticles. ${ }^{20,21}$

These studies addressed different kinds of high-interest nanomaterials such as nanotubes, ${ }^{3}$ quantum dots, ${ }^{1,2}$ nano-wires, ${ }^{4}$ and noble metal nanoparticles. ${ }^{5-21}$ Due to tremendous progress in metallic colloidal chemistry synthesis, ${ }^{22,23}$ a wide vari-ety of shapes have been investigated: spheres, $^{5,6,13,18}$ rods, $^{11,12}$ prisms and triangles, ${ }^{7,10,15,16}$ dimers, $^{17}$ cubes, $^{19}$ and bipyramids. ${ }^{9}$ These measurements were performed both on ensembles of particles and more recently on single particles. $4,12-15,17$ These vibrations have been investigated by different optical techniques: Raman scattering ${ }^{2,6,8}$ and time-resolved femtosecond spectroscopy principally. ${ }^{1,3-5,7-21} \mathrm{Me}-$ tallic systems represent a large part of such studies since they present a very specific feature in their optical properties, the surface plasmon resonance (SPR), which is very sensitive to the size, shape, and environment of the particles and is directly involved in the detection of particle vibrations. The comprehension of these vibrations is of major interest in the nanoscience field since they address both fundamental and applied issues. They play an important role in the energy transfer from a nanosystem to its environment, and the study of the damping mechanism remains a hot topic. ${ }^{9,13-15,17}$ Moreover, the high-frequency range addressed by this kind of nanosystem (10 $100 \mathrm{GHz}$ ) is of major interest for applications in the nanobalance domain. ${ }^{21,24}$ In this framework, hybrid nanoobjects formed by two or more material components of different nature offer a wide range of possibilities for the development of novel nanosystems. Indeed, it is now possible to envisage the control and tuning of their individual properties and get new functionalities that can fit specific needs. In this context, many metallic systems investigated presented interesting behavior in ensemble measurements but revealed a great reduction of
* Address correspondence to julien.burgin@u bordeaux1.fr. 
vibration damping due to the removal of inhomogeneous size effects when investigated at the singleparticle level. ${ }^{12-15}$ Meanwhile, the gold bipyramid has proven to be a very interesting system because it has a very narrow size distribution and allows ensemble measurements with low damping rates, opening up many possibilities in terms of practical applications. ${ }^{9,25,26}$ These nanosystems are also promising because they present a very easily tunable SPR in the infrared adapted to biosensing applications, ${ }^{27}$ and their sharp apex is expected to generate great local field enhancement. ${ }^{28}$

We present here a comprehensive study on coherent vibrations of core gold bipyramids in water and gold silver core shell bipyramids based on studied core samples. We addressed extensional and radial vibrations as a function of the size of the particles, and we also present a study of the evolution of the mode amplitude under silver deposition for core shell bipyramids. Our results are also confronted with finite-element analysis calculations.

\section{RESULTS AND DISCUSSION}

Figure 1a shows the extinction spectra of a gold core sample and three gold silver core shell samples. The core spectrum presents two surface plasmon resonances, one centered near $800 \mathrm{~nm}$ corresponding to the longitudinal resonance of gold bipyramids and another, near $515 \mathrm{~nm}$, corresponding to SPR of gold spheroids, a coproduct of the synthesis. ${ }^{27,29}$ As expected, the deposition of silver onto the gold core bipyramids induces a blue shift of both resonances. ${ }^{30,31}$ Figure $1 \mathrm{~b}$ is a TEM image of gold core bipyramids where we clearly see their anisotropic shape, which one can simply model by a bicone or a bicone with a rounded tip with a typical curvature radius of $5 \mathrm{~nm}$. Figure $1 \mathrm{c}$ is a TEM image of silver gold core shell bipyramids where an significant quantity of silver has been deposited (corresponding to $1 \mathrm{~mL}$ in our protocol). We clearly see that the silver envelope presents a kind of ellipsoidal or cylindrical shape. These nanostructures have been studied with 3D TEM, and it has been shown that the silver thickness is not constant and varies between 0 and $6 \mathrm{~nm}$ typically. ${ }^{31}$ This is why we prefer to consider the injected silver quantities in milliliters rather than geometric arguments. In our numerical simulations, according to the measured 3D shapes, ${ }^{31}$ we decided to model the core shell bipyramids by a bicone surrounded by a shell defined by a parametric function yielding intermediate shapes between a bicone $(\gamma=0)$ and an ellipsoid $(\gamma=2)$ (see Figure 1d and eq 2).

Time-resolved experiments are performed on several samples of gold core bipyramids with different lengths and diameters measured from TEM microscopy. We use a degenerate configuration with the same pump and probe wavelength. The wavelength is chosen in the vicinity of the bipyramids' SPR in order to be sensitive to their acoustic vibrations. Indeed, the
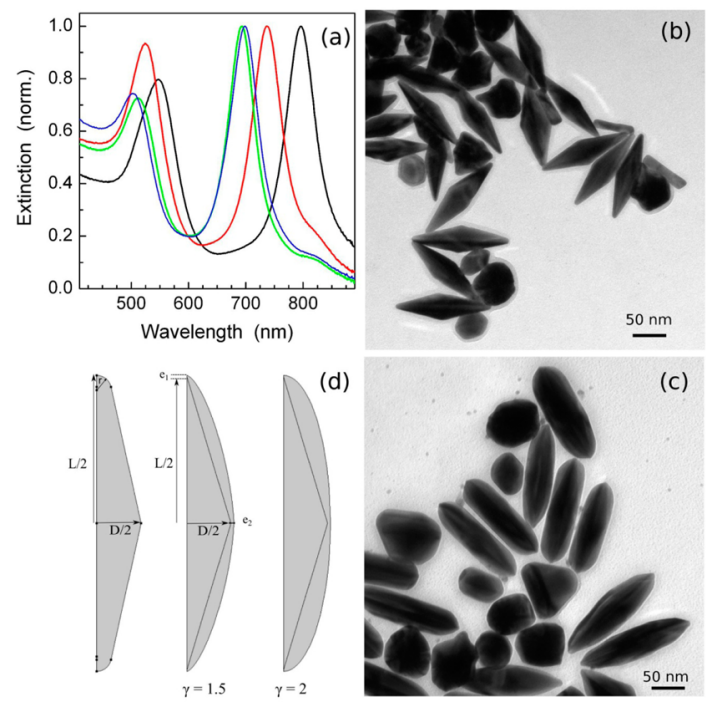

Figure 1. (a) Extinction spectra of Au core bipyramids (black line) and Au@Ag core shell bipyramids for different silver addition (increasing silver quantity: red, green, blue). (b) TEM image of Au core bipyramids. (c) TEM image of Au@Ag core shell bipyramids. (d) Transverse geometry of the particles used for finite element analysis in $2 \mathrm{D}$ axisymme try. From left to right: Core bicone with a rounded tip (curvature radius $r$ ); core bicone (with $r=0 \mathrm{~nm}$ ) surrounded by a shell with the shape parameter $\gamma=1.5$ and 2 (eq 2). The longitudinal and lateral offset of the shell are $e_{1}$ and $e_{2}$.

ultrashort pump pulse induces a thermal expansion that launches acoustic vibrations, and the delayed probe pulse probes these vibrations that affect the SPR. ${ }^{5}$ Figure 2 shows a typical signal obtained in gold core bipyramids (reference core sample): during the first picoseconds, we observe the electronic response and energy transfer to the lattice, ${ }^{32}$ while for longer probe delay we clearly see strong oscillations that correspond to radial and extensional vibrations. To extract the characteristics of the different vibration modes, we perform numerical fits using the following equation:

$$
f(t)=\sum_{i} A_{i} \mathrm{e}^{-t / \tau_{\mathrm{i}}} \cos \left(\frac{2 \pi}{T_{i}} t+\varphi_{i}\right)+B \mathrm{e}^{-t / \tau_{\mathrm{bg}}}
$$

where $A_{i}, T_{i}, \tau_{i}$, and $\varphi_{i}$ correspond to the amplitude, period, damping time, and phase of the mode $i$, and $B$ and $\tau_{\text {bg }}$ correspond to the amplitude and characteristic time of the background signal related to energy transfer to the medium. ${ }^{33}$ The red line in Figure 2 corresponds to a fit using two modes. The principal one, with a period measured at $T_{0}=53.8 \mathrm{ps}$, has already been observed and associated with the fundamental extensional mode of the bipyramids; ${ }^{9}$ the second one, with a period around $T_{1}=25.5 \mathrm{ps}$, is observed for the first time in bipyramids: we associated it with the firstharmonic extensional mode. This mode is quite weak but present in our experimental data and has to be considered in order to get a good fit. The first-harmonic mode observed here is weak in nanorods but has already been observed in nanoprisms. ${ }^{15}$ These two measured periods are in very good agreement with 


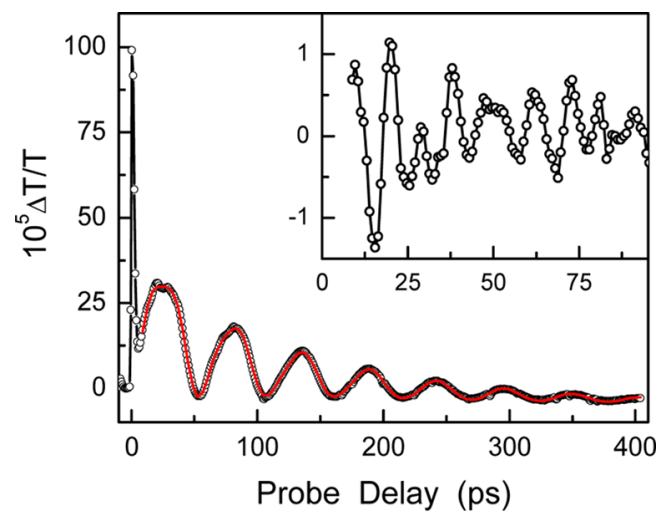

Figure 2. Time dependence of the relative transmission changes $(\Delta T / T)$ measured in an ensemble of gold core bipyramids with a average length of $78 \mathrm{~nm}$ and diameter of $28 \mathrm{~nm}$ (black line and circles) and numerical fit with the sum of two exponential decayed sinusoids (red line). Inset: Residual oscillating component from subtraction of the two modes fit to the data. These oscillations are due to radial vibrations.

numerical calculation: the fundamental mode period is found to be 53.4 ps, and its first-harmonic 26.1 ps. These calculations are done with finite-element analysis software (COMSOL) in 2D axisymmetry using the elastic constants of bulk polycrystalline gold (Young's modulus $E=78.5 \mathrm{GPa}$, Poisson's ratio $v=0.42$, and density $\rho=19300 \mathrm{~kg} \mathrm{~m}^{-3}$ ). We used polycrystalline elastic constants since they are very close to the ones associated with the longitudinal axis [110] of the particles. ${ }^{11,12,31}$ To study the validity and robustness of this approach, we performed several measurements with different particle lengths (from 49 to $170 \mathrm{~nm}$ ) and diameters (from 20 to $40 \mathrm{~nm}$ ) to study the evolution of these high-frequency modes. Taking advantage of the tunability of the laser source, we adapted the probe according to the position of the surface plasmon resonance wavelength for every sample. We report the measured periods for both the fundamental and first-harmonic period in Figure 3. The measured periods are found to be linearly dependent with their length, as measured in nanorods. ${ }^{11,12,34}$ To associate the measured periods with these modes, we perform several numerical calculations varying the length of particles. We first verify that the measured periods are consistent with the dimension and the shape of the nano-objects, calculating the periods for a cylinder and a bicone. These results are shown in Figure 3 (dotted and dashed lines) and border our measurements for both modes. This was expected since the elongated particles represented in Figure 1d have an intermediate shape between a cylinder (infinite tip curvature radius) and a bicone (zero tip curvature radius). To analyze our data, we calculate the periods as a function of the length of an average bipyramidal particle corresponding to our samples (considering the bipyramids as bicones with a diameter $D=40 \mathrm{~nm}$ and a rounded tip with a curvature radius $r=6 \mathrm{~nm}$ ). The results are in very good agreement, even for extreme sizes.

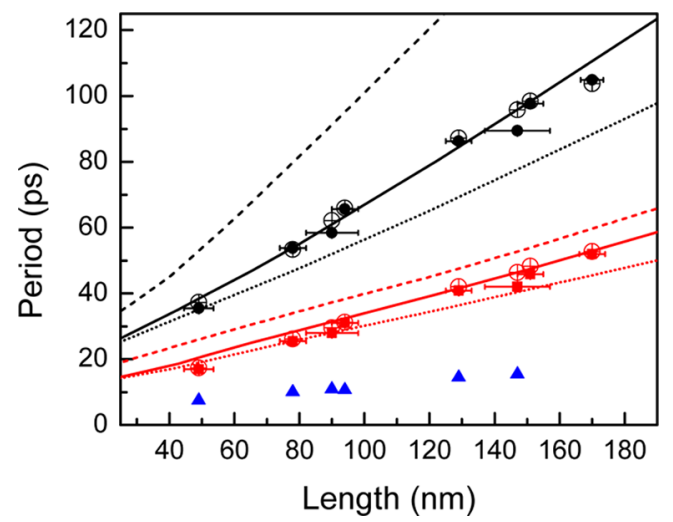

Figure 3. Periods of the vibrational modes measured for several different samples of gold core bipyramids as a function of their length. The circles correspond to the fundamental longitudinal mode $\left(T_{0}\right)$, squares correspond to the first long itudinal mode harmonic $\left(T_{1}\right)$, and triangles to the fundamental radial mode $\left(T_{\mathrm{rad}}\right)$. The full lines correspond to computed periods for a realistic bipyramidal shape (diameter $D=$ $40 \mathrm{~nm}$ and a tip curvature radius $r=6 \mathrm{~nm}$ ); the dotted and dashed lines correspond to computed periods for a biconical shape $(D=40 \mathrm{~nm})$ and a cylinder $(D=40 \mathrm{~nm})$, respectively (black lines correspond to $T_{0}$ and red lines to $T_{1}$ ). The crossed circles correspond to numerical simulation using dimensions extracted from TEM analysis for each sample.

From this numerical analysis, we can derive qualitative linear laws between the period and length for each shape: $T=\alpha_{\text {shape }} L$. For the fundamental mode, the values are $\alpha_{\text {bipyr }}=0.64 \mathrm{ps} / \mathrm{nm}$ for bipyramids, $\alpha_{\text {bicone }}=$ $0.49 \mathrm{ps} / \mathrm{nm}$ for bicones, and $\alpha_{\text {cyl }}=0.99 \mathrm{ps} / \mathrm{nm}$ for cylinders. For the latter, both analytical calculations and numerical simulations predict this result. ${ }^{34}$ For specific shapes such as bicones and bipyramids, finite-element solutions are a simple way to get the size dependence of the periods. To go further, we perform realistic simulations for every sample, designing them with dimensions extracted from TEM analysis. These calculations are represented by crossed circles and are in very good agreement with experimental data. This fine analysis associating time-resolved experiments and finite-element analysis is the first one dealing with both fundamental and first-harmonic mode and their size dependence.

We also investigated the damping time of the fundamental mode. Since our measurements are performed on a large collection of particles, the measured damping time $\tau_{i}$ with eq 1 includes an inhomogeneous contribution due to the size distribution of the studied samples. To get rid of this problem and extract the intrinsic damping time $\tau_{\mathrm{h}}$, we perform a numerical analysis already presented in previous studies for samples with a narrow size distribution, which is possible in this study since standard deviations are smaller than $5 \%$ (see Supporting Information). ${ }^{9,35}$ We thus measure the quality factor of the fundamental mode $Q=\pi\left(\tau_{\mathrm{h}} / T\right)$. We found that it is independent of the length of the bipyramids, and its average value is $Q=18 \pm 4$. This result confirms previous measurements for the same particles of one particular length ${ }^{9}$ and 
is in good agreement with measurements performed on single gold nanosystems where they measured quality factors in water around 26 in gold nanowires ${ }^{4}$ and 28 in nanorods. ${ }^{12}$ These small differences probably come from surface internal defects in bipyramids that may be more important due to their complex crystallographic structure. ${ }^{31}$ These relatively high quality factors offer large possibilities in the field of nanosensors and nanobalances for metallic nanostructures since their vibration frequencies are in the ultrahigh frequency range.

A better look at the data in Figure 2 reveals that the two-mode fit does not mimic the signal perfectly for delays around 20 ps. This kind of phenomenon is observed when short period modes are present. These modes are generally associated with the vibration along the shortest dimensions of the particle. They have already been observed in nanoprisms, ${ }^{15}$ nanocolumns, ${ }^{8}$ and nanorods. ${ }^{12}$ To understand this feature, we can consider the residual signal originating from the difference between the experimental data and a two-mode fit using eq 1. The results are shown in the inset of Figure 2. We clearly see residual oscillations. However, to estimate accurately the vibrational period, we perform a fit on the whole data set using three modes (eq 1). The period of the weakest mode is found to be $10.1 \mathrm{ps}$. Finite element analysis allows us to associate it with the fundamental

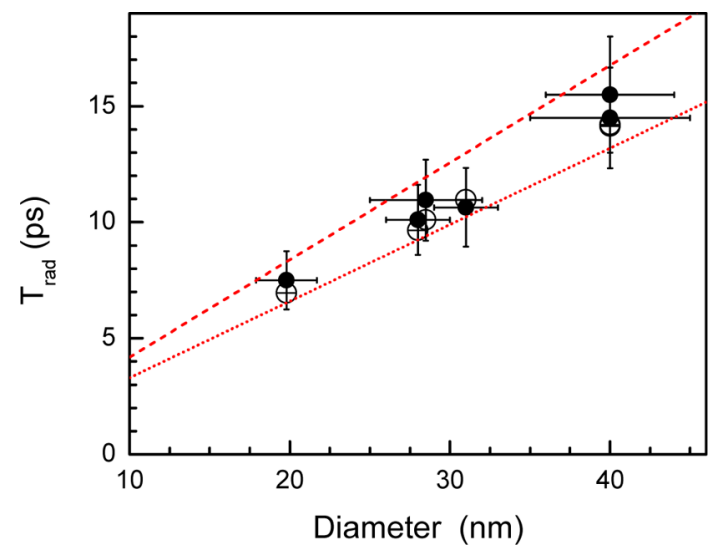

Figure 4. Periods of the radial mode measured for several gold core bipyramids as a function of their diameter. The dotted and dashed lines correspond to computed periods for a biconical particle (aspect ratio $L / D=3$ ) and a cylinder $(L / D=3)$, respectively. The crossed circles correspond to numerical simulation using dimensions extracted from TEM analysis for each sample. radial mode of the bipyramids. The calculated period (9.8 ps) is in good agreement with the experimental value. This vibrational mode is strong and easily observable in nanorods since the radial and longitudinal vibrations are well decoupled. ${ }^{12,34}$ This is not the case for bipyramids, where this mode is weak but observable in our setup, as we can see it in the inset of Figure 2. Figure 4 summarizes the evolution of its period as a function of the bipyramid diameter. We find a linear dependence with the diameter. This result is expected because this behavior is observed and predicted in nanorods modeled as cylinders. ${ }^{12,34}$ To realize a quantitative analysis of this set of data, we perform finiteelement analysis analogous to the previous one concerning extensional modes. We compute the period of the first radial mode in cylinders (dashed line in Figure 4) and in bicones (dotted line in Figure 4). From this numerical analysis, we can then derive qualitative linear laws between period and length for each shape: $T_{\text {rad }}=$ $\beta_{\text {shape }} D$. The values are found to be $\beta_{\text {bicone }}=0.31 \mathrm{ps} / \mathrm{nm}$ for bicone and $\beta_{\text {cyl }}=0.42 \mathrm{ps} / \mathrm{nm}$ for cylinders for an aspect ratio $L / D$ fixed at 3 . We also perform calculations for more realistic shapes, bicones with a rounded tip (Figure 1d), and the results are found to be intermediate between bicone and cylinder. We find a good agreement with our experimental data. For the sake of simplicity, we use only one mode to fit the radial vibrations, but finer experiments (on single particles, for example) could reveal more complex vibrational features in these particles. Indeed, it should be interesting to investigate the potential presence of hybridized radial modes that can exist in systems with complex transverse symmetry. Note that hybridized modes have been observed in single suspended gold nanowires. ${ }^{4}$ We also verified that the use of bulk polycrystalline elastic constants do not affect the accuracy of our calculations. Indeed, with an average speed of sound for the transversal directions of the bipyramids ([100] and [110]) the computed periods are slightly modified (around 4\%).

Another aspect of our investigations deals with the modes' amplitudes. We take care to perform these experiments on the red side of the SPR (at its inflection point), where the amplitudes of radial and extensional modes are locally independent of the probe wavelength. ${ }^{12}$ The amplitude ratios are merely constant for the whole batch of gold bipyramids. Fitting the data

TABLE 1. Mode Amplitude Ratios Measured on Core and Core Shell Particles and Ratio Normalized over the Ratio Measured in the Core Particle ${ }^{a}$

\begin{tabular}{|c|c|c|c|c|c|c|}
\hline & $A_{1} / A_{0}$ & $A_{\mathrm{rad}} / A_{0}$ & $A_{\mathrm{rad}} / A_{1}$ & $\frac{\left(A_{0} / A_{1}\right)^{\mathrm{CS}}}{\left(A_{0} / A_{1}\right)^{\mathrm{core}}}$ & $\frac{\left(A_{\text {rad }} / A_{0}\right)^{c s}}{\left(A_{\text {rad }} / A_{0}\right)^{\text {core }}}$ & $\frac{\left(A_{\text {rad }} / A_{1}\right)^{\mathrm{cs}}}{\left(A_{\mathrm{rad}} / A_{1}\right)^{\mathrm{core}}}$ \\
\hline core & $0.25 \pm 0.03$ & $0.094 \pm 0.015$ & $0.37 \pm 0.06$ & 1 & 1 & 1 \\
\hline CSa & $0.21 \pm 0.03$ & $0.12 \pm 0.02$ & $0.59 \pm 0.08$ & $1.2 \pm 0.2$ & $1.3 \pm 0.4$ & $1.6 \pm 0.5$ \\
\hline $\mathrm{CSb}$ & $0.054 \pm 0.006$ & $0.18 \pm 0.02$ & $3.37 \pm 0.47$ & $4.6 \pm 1.0$ & $1.9 \pm 0.5$ & $9.1 \pm 2.7$ \\
\hline $\mathrm{CSC}$ & $0.039 \pm 0.006$ & $0.29 \pm 0.03$ & $7.4 \pm 1.2$ & $6.4 \pm 1.6$ & $3.1 \pm 0.8$ & $20 \pm 6$ \\
\hline
\end{tabular}

${ }^{a}$ The amplitude values are extracted from fits using eq 1. 
TABLE 2. Ratio of the Excitation Amplitudes of Several Modes for Core Bicone (a), Pentagonal Bipyramid (b), and Three Core Shell Particles Based on the Pentagonal Core with the Shells Made with an Ellipsoid (c), with the $\gamma$ Parameterized Shape Using $\gamma=3$ (d), or with a Rounded Cylindrical Shape (e) ${ }^{a}$

\begin{tabular}{|c|c|c|c|c|}
\hline Particle shape & $\mathrm{A}_{1} / \mathrm{A}_{0}$ & $\frac{\left(\mathrm{A}_{0} / \mathrm{A}_{1}\right)^{\mathrm{CS}}}{\left(\mathrm{A}_{0} / \mathrm{A}_{1}\right)^{\mathrm{core}}}$ & $\mathrm{A}_{\text {rad }} / \mathrm{A}_{0}$ & $\frac{\left(\mathrm{A}_{\mathrm{rad}} / \mathrm{A}_{0}\right)^{\mathrm{CS}}}{\left(\mathrm{A}_{\mathrm{rad}} / \mathrm{A}_{0}\right)^{\mathrm{core}}}$ \\
\hline (a) & 0.188 & $\mathrm{X}$ & 0.668 & $\mathrm{X}$ \\
\hline (b) & 0.186 & 1 & 0.342 & 1 \\
\hline (c) & 0.181 & 1.04 & 0.838 & 2.45 \\
\hline (d) & 0.115 & 1.62 & 0.646 & 1.89 \\
\hline (e) & 0.066 & 2.8 & 0.613 & 1.79 \\
\hline
\end{tabular}

${ }^{a}$ The core dimensions are $L=78 \mathrm{~nm}$ and $D=28 \mathrm{~nm}$, and the shell is $79 \mathrm{~nm}$ long $\left(e_{1}=0.5 \mathrm{~nm}\right)$ and $34 \mathrm{~nm}$ wide $\left(e_{2}=3 \mathrm{~nm}\right)$.

with a three-mode function allows us to find $A_{1} / A_{0} \approx$ $0.25, A_{\text {rad }} / A_{0} \approx 0.094$, and $A_{\text {rad }} / A_{1} \approx 0.37$ (Table 1). We observe a strong domination of the vibrational response by the fundamental mode. This result is to be compared with the vibrations measured in nanorods where the radial vibrations are strong and present nearly the same amplitude as extensional vibrations ${ }^{12}$ (for a probe wavelength on the red side of the SPR) and where the first harmonic is very difficult to observe. These differences are due to the shape of the particles. The expected amplitudes are difficult to predict, even for simple geometries, because both excitation mechanism and detection process in the vicinity of the SPR have to be modeled precisely. ${ }^{36}$ Meanwhile, we can compute the excitation amplitudes for several core particle shapes in 3D geometry (see Supporting Information). We find that for $3 \mathrm{D}$ rounded tip bicones and for $3 \mathrm{D}$ pentagonal rounded bipyramids, $A_{1} / A_{0} \approx 0.19$, which is in fair agreement with the measured values (Table 2). For the radial mode, we found $A_{\text {rad }} / A_{0} \approx 0.67$ and 0.34 for a bicone and a bipyramid, respectively, values larger than the measured one $(\sim 0.1)$. First, note here that the more realistic pentagonal section has a strong effect on the radial mode amplitude, a reduction by a factor 2 . Since the section of the particle plays an important role, we can wonder if a finer description taking into account the twisted shape of the bipyramids and/or its complex transverse profile could explain this discrepancy. ${ }^{31,37}$ Single-particle measurement would be necessary to address these points. Second, we must keep in mind that these calculations do not take into account the

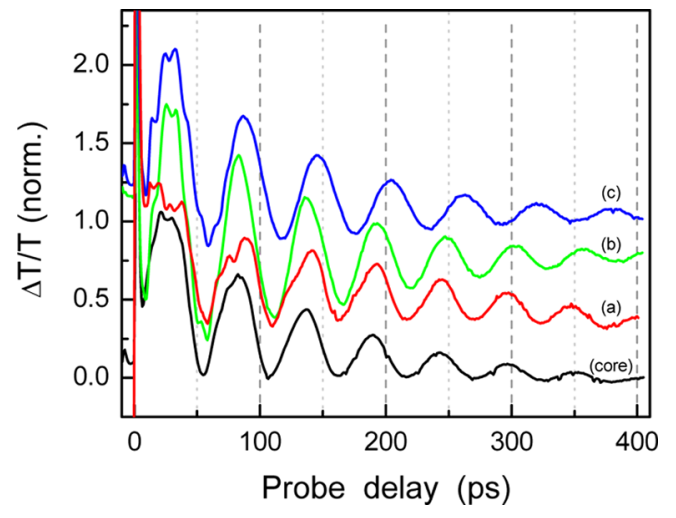

Figure 5. Time dependence of the relative transmission changes $(\Delta T / T)$ measured in Au core bipyramids with a length of $78 \mathrm{~nm}$ and a diameter of $28 \mathrm{~nm}$ (black line) and Au@Ag core shell nanoparticles (sample CSa, red line; sample CSb, green line; sample CSc, blue line). For more clarity, the three colored curves are arbitrary shifted.

detection efficiency. This effect can be estimated for spherical particles ${ }^{36,38}$ and has been found to be quite important and dependent on the displacement field of the considered mode (see Supporting Information). ${ }^{38}$ Since the radial and extensional modes have very different displacement fields, we can reasonably associate this discrepancy with the detection efficiency. Finally, the calculation of the excitation amplitudes in a rounded cylinder mimicking nanorods shows that the harmonic mode is weakly excited $\left(A_{1} / A_{0} \approx 0.006\right)$, while the radial mode is strongly excited $\left(A_{\mathrm{rad}} / A_{0} \approx 0.68\right)$, which is in good agreement with measurements 

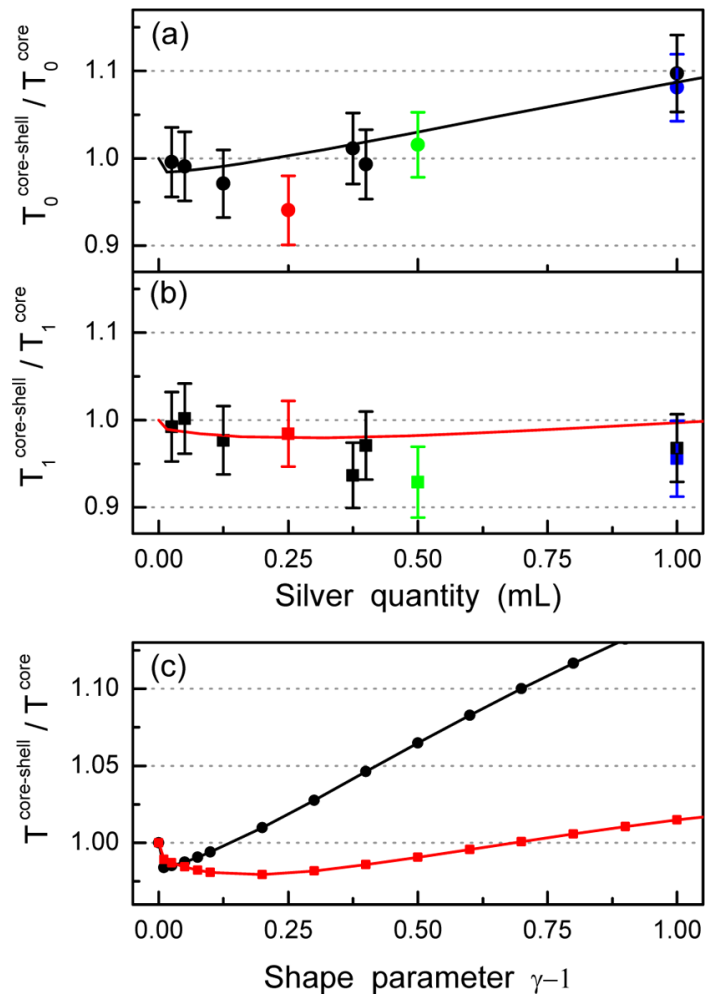

Figure 6. Measured vibrational periods for Au@Ag core shell samples, normalized on the measured period for $\mathrm{Au}$ core particles as a function of quantity of silver added during the synthesis (fundamental mode (a), first harmonic (b)). The three samples CSa, CSb, and CSc are represented in red, green, and blue, respectively. The black symbols corre spond to other core shell samples based on different cores. (c) Computed periods for a biconical core shell particle as a function of the shape parameter $\gamma$ from eq 2 (black line, fundamental mode $T_{0}$; red line, first harmonic $T_{1}$ ). The periods are normalized over the core periods, corre sponding to $\gamma=0$. The computed periods are superimposed with experimental data on (a) and (b) using the following calibration with an equivalent volume $V_{\mathrm{eq}}=\left(\begin{array}{ll}\gamma & 1\end{array}\right) / 0.6$.

obtained by Zijlstra et al. (see Supporting Information). ${ }^{12}$ We clearly see here that the initial condition (isotropic dilatation) has a bigger projection over the first-harmonic mode for a bipyramidal shape than a cylindrical one.

After studying the vibrations of gold core bipyramids, we focused on the evolution of the vibrational modes under silver deposition. Similar experiments are performed on Au@Ag core shell bipyramids synthesized from different gold core bipyramid samples already studied. Figure 5 shows the measured $\Delta T / T$ signals on the reference gold core sample $(L=78 \mathrm{~nm}$, $D=28 \mathrm{~nm}$ ) and three core shells based on it. The core shell CSa, CSb, and CSc samples correspond to an injection of $0.25,0.5$, and $1 \mathrm{~mL}$ of $1 \mathrm{mM} \mathrm{AgNO}_{3}$ solution, respectively.

After a similar fast transient signal we observe pronounced vibrations for every sample and a phase shift between them for longer delays ( $\pi$ phase shift for $\mathrm{CSc}$ at $350 \mathrm{ps}$ ), proving that the periods are affected by silver deposition. Moreover, for a probe delay between 25 and 75 ps, we see that short period vibrations are strongly modified, principally their amplitude. These short period vibrations, which have been associated with the radial mode, are then significantly excited when the silver quantity deposited increases. First, we monitor the evolution of the vibrational periods under silver deposition, performing experiments with several silver quantities deposited on several gold core bipyramid samples. The results obtained on $\mathrm{CSa}, \mathrm{CSb}, \mathrm{CSc}$, and core shell samples based on different gold cores are summarized in Figure 6, where the periods of the fundamental mode and its first harmonic (normalized over the measured period of the corresponding core sample) are plotted as a function of the silver quantity injected during the synthesis. We observe a rich behavior for both modes. For small amounts of deposited silver, $T_{0}$ decreases rapidly down to $5 \%$ of the core period and then finally increases for large amounts of silver, up to $10 \%$. For small amounts of silver deposited, $T_{1}$ gently decreases down to $8 \%$ and then begins a slight rise for larger silver deposition. This asymmetric behavior can originate from the specific silver deposition that occurs here preferentially on the lateral facets with a small amount of silver on the tip. By the way, the average aspect ratio of the particles barely decreases while silver mass is deposited onto them and since the two modes do not have the same lateral and longitudinal displacements; their periods are affected differently by silver deposition. To interpret these observations, we perform finite-element analysis of the mechanical vibrations with biconical core shell particles sketched in Figure 1d. Taking account of the shape extracted from 3D TEM analysis of this kind of object ${ }^{31}$ and in order to get a scalable and simple shape factor mimicking the silver quantity deposited, we design the shell as follows. The simulations are performed in the axisymmetrical configuration, where a 2D drawing is sufficient for the calculation. Here, the core is designed with two triangles, yielding a simple bicone, while the shell is delimited by a radius $\rho$ depending on the altitude $z$ varying between $L / 2$ and $L / 2$ :

$$
\rho(z)=e_{2}+\frac{D}{2}\left(1\left(\frac{z}{\frac{L}{2}+e_{1}}\right)^{\gamma}\right)^{1 / \gamma}
$$

where $e_{1}$ and $e_{2}$ are longitudinal and radial offsets, respectively (Figure 1d), and $\gamma$ is a shape parameter varying between extreme values 1 and 2 . The value $\gamma=1$ generates a bicone slightly bigger than the core (assuming $e_{1}>0$ and $e_{2}>0$ ), corresponding to a slight silver deposition. The value $\gamma=2$ creates an elliptical shell shape surrounding the core, corresponding to a substantial silver deposition. We use typical values measured in TEM for $e_{1}$ and $e_{2}\left(e_{1}=0.5 \mathrm{~nm}\right.$ and $\left.e_{2}=1 \mathrm{~nm}\right)$ and $L=100 \mathrm{~nm}$ and $D=30 \mathrm{~nm}$ for the core bipyramids to calculate the evolution of the periods $T_{0}{ }^{\mathrm{cs}}$ and $T_{1}{ }^{\mathrm{cs}}$ as a function of $\gamma$. In Figure $6 c$, we observe that for $1<\gamma<1.15$ the ratio $T_{0}^{\mathrm{cs}} / T_{0}^{\text {core }}$ is inferior to 1 and then increases 
linearly for $\gamma>1.2$ up to the value 1.15 for $\gamma=2$. Qualitatively, this non-monotonic behavior is in good agreement with the measured periods. The calculated behavior is intermediate to the one calculated for pure $\mathrm{Au}$ or Ag using the same parametrized shape (see Supporting Information). Concerning the first-harmonic mode, the ratio $T_{1}^{\mathrm{cs}} / T_{1}$ core is found to be less than 1 and decreases for $1<\gamma<1.2$, increases for $\gamma>1.2$, and becomes greater than 1 if $\gamma>1$.7. This behavior is partially observed in our experimental data. Experimentally, the ratio $T_{1}{ }^{\mathrm{cs}} / T_{1}$ core is less than 1 for low silver deposition and presents a slight increase for substantial silver deposition. We have not been able to observe the $T_{1}^{\mathrm{cs}} / T_{1}{ }^{\text {core }}>1$ regime because of the low stability of highly silvered samples. By the way, according to these observations we can assume that our measurements on samples with the largest amount of silver correspond roughly to $\gamma \approx 1.6$ in the framework of these $2 \mathrm{D}$ simulations. Both the absolute increase in $T_{0}^{\mathrm{cs}} / T_{0}$ core up to 1.1 and the increase of $T_{1}{ }^{\mathrm{cs}} / T_{1}$ core near 1 for the highly silvered sample lead us to this assertion. Even if these calculations were not performed with a realistic rounded tip bicone for the core particle (in order to address the $1<\gamma<2$ range), we superimposed them with our experimental data (Figure $6 a$ and b). To do so, we calibrated the abscissa axis by defining an equivalent volume $V_{\text {eq }}(\gamma)=\left(\begin{array}{ll}\gamma & 1\end{array}\right) / 0.6$. Consequently, $\gamma=1$ corresponds to an equivalent volume $V_{\text {eq }}=0 \mathrm{~mL}$ and $\gamma=1.6$ corresponds to a volume $V_{\text {eq }}=1 \mathrm{~mL}$. The results are in good qualitative agreement, but one would need a better correspondence between the real and computed shape to make quantitative conclusions. To go further, the influence of $e_{1}$ and $e_{2}$ could be investigated because their effect on the calculated periods is quite important, particularly for low silver quantities where $T_{0}^{\mathrm{Cs}}$ / $T_{0}{ }^{\text {core }}<1$. A more quantitative analysis of these core shell nanostructures would necessitate measurements on single particles associated with exact 3D TEM characterization coupled with finite-element analysis simulations.

We now focus on another aspect induced by silver deposition onto these gold bipyramids: the evolution of the modes' amplitudes. The most important effect, previously mentioned, is the important increase of the radial mode amplitude under silver deposition. We measure the amplitudes of each mode in core shell samples and calculate the ratios $A_{\text {rad }} / A_{0}, A_{1} / A_{0}$, and $A_{\mathrm{rad}} / A_{1}$. To monitor their evolution under silver deposition, we normalize them over the ratio measured in the corresponding gold core sample. The results are presented in Table 1, and a part of them are shown in Figure 7 for our reference sample set made of three core shell samples CSa, CSb, and CSc. First, we observe that the ratio $A_{0} / A_{1}$ increases with silver deposition, up to a factor of 6.4. This is due to a strong quenching of $A_{1}$, the harmonic mode amplitude. At the same time, we observe an increase of $A_{\text {rad }}$ over both $A_{0}$ and $A_{1}$. For sample CSc, the ratio $A_{\text {rad }} / A_{1}$ of 20 is due to both an increase of $A_{\text {rad }}$ and quenching of $A_{1}$.

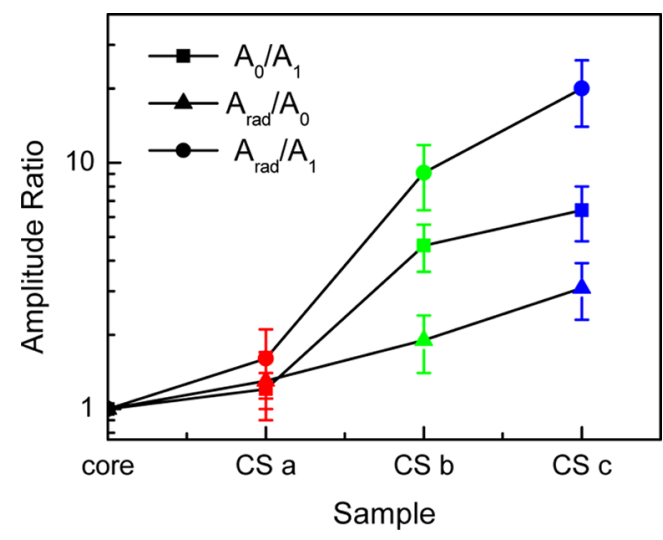

Figure 7. Amplitude ratios measured on core shell parti cles, normalized over the ratio measured in the core parti cle. The amplitude values are extracted from fits using eq 1. The circles correspond to $A_{\text {rad }} / A_{1}$, triangles correspond to $A_{\text {rad }} / A_{0}$, and squares correspond to $A_{0} / A_{1}$.

We also notice the large increase of the ratio $A_{\text {rad }} / A_{0}$ up to 3.1 in sample CSc, clearly showing that the radial mode is more excited in core shell than in core particles. To understand this behavior, we perform numerical calculation of the excited amplitude for core shell particles of several geometrical shapes. As previously discussed, the transverse shape has an important impact on the amplitudes; we thus calculate them for core particles with a pentagonal symmetry and a rounded tip (length $L=78 \mathrm{~nm}$, diameter $D=28 \mathrm{~nm}$, and tip radius $r=5 \mathrm{~nm}$ ), to approximate as best as we can the particle shape. The results are presented in Table 2. With an ellipsoidal silver shell, we find an increase of 1.04 and 2.45 for $A_{0} / A_{1}$ and $A_{\text {rad }} / A_{0}$, respectively. Using this geometry, our calculation predicts the increase of the radial mode correctly but predicts less accurately the quenching of the first-harmonic mode. To go further, we can implement the already used parametrization $\rho(z)$ (eq 2) in three dimensions using $\gamma=3$ in order to design a more rounded shell. We obtain a stronger quenching of the first harmonic (factor 1.62) but a slighter increase of the radial mode (factor 1.89). Meanwhile, the calculated excitation amplitudes using this shape reproduce the trend observed experimentally. Finally, we can also calculate the amplitudes using a cylindrical shape ended by two spheres. This extreme shape yields an important quenching of the first-harmonic mode, by a factor of 2.8. Note here that the value $A_{1} / A_{0}=0.066$ is thus very close to the one calculated in a pure gold rounded cylinder (0.058). This confirms the experimental observations on core bipyramids, nanorods, ${ }^{12}$ and core shell bipyramids; the transformation of a bipyramid into a more cylindrical shape induces a quenching of the first-harmonic mode. Meanwhile, even if we do not predict exactly the ratios' evolution, their absolute values are close (the measured value of $A_{1} / A_{0} \approx 0.04$ is comparable to the calculated value 0.066 ). Concerning the radial mode, the rounded cylindrical shell yields an 
enhancement factor of 1.8, which is in fair agreement with experimental values. As previously mentioned, we think that the detection efficiency depends strongly on the particle shape and is responsible for the deviations observed between experiments and simulations (see Supporting Information). For the radial mode, these calculations underestimate the experimental enhancement: we can thus suppose that the radial mode detection efficiency increases as far as silver is deposited, and the particle looks cylindrical. This could be also the case in nanorods, where $A_{\mathrm{rad}} / A_{0} \approx 1$ in experiments, ${ }^{12}$ whereas $A_{\mathrm{rad}} / A_{0} \approx 0.68$ in simulations (see Supporting Information). For the firstharmonic mode, these simulations underestimate the experimental quenching, which suggests that the detection efficiency of this mode is not constant and drops as far as silver is deposited.

Then, monitoring the evolution of amplitudes yields information on mass deposition. By the way, the evolution of $\left(A_{\mathrm{rad}} / A_{0}\right)^{\text {core-shell }} /\left(A_{\mathrm{rad}} / A_{0}\right)^{\text {core }}$ from 1 to $1.3,1.9$, and 3.1 in this experiment can be related to an approximate mass deposition of 40,80 , and 160 attograms per particle, respectively. This sensitivity is not the best one can get for mass sensing, ${ }^{39}$ but these nanosystems present interesting properties: local sensing at the nanometric scale, versatility in solid-state deposition sensing, and fluid environment capabilities. Moreover, since both periods and amplitudes are sensitive to mass deposition on the facets, one can imagine a cross-checking procedure based on several parameters (relative amplitudes and periods of three modes) that are very sensitive to the mass deposition and their localization, in order to get rid of experimental artifacts based on a single frequency measurement. These results give a new perspective for metallic mass sensing systems and nanobalances for solid-state materials. The extension of this study to protein sensing will probably face two issues. First, if we roughly consider that mass is the relevant parameter, the detection limit of 40 ag corresponds to 80 proteins of $300 \mathrm{kDa}$, which is far from the single-protein detection level reached using surface plasmon resonance. ${ }^{40,41}$ Second, this would require a new modeling dealing with localized adsorption of a soft material on the particles. Such modeling and experimental work should focus on the effect of protein adsorption on the damping of vibrations and particularly the impact of the environment viscosity and the protein geometry on the detection limits. ${ }^{42}$

\section{CONCLUSION}

To conclude, we investigated the vibrational modes of bare gold nanobipyramids and gold silver core shell bipyramids using a time-resolved femtoscecond technique. We measured and simulated the evolution of two extensional modes and a radial mode in gold bipyramids as a function of their size. The results are in very good agreement with finite-element analysis calculations of the periods and amplitudes. We also measured the quality factor of the fundamental mode and found it was independent of the particle size. In core shell nanosystems, we monitored the evolution of the periods of the two extensional modes, which presented a complex behavior. A simple geometry and finite-element analysis were found to be in good qualitative agreement with the measurements taking account of the particular shape of these core shell systems where the silver is preferentially deposited on the facets. Finally, we monitored the evolution of the amplitude of the three modes and found a strong enhancement of the breathing mode under silver deposition. This behavior has been compared to finite-element analysis calculations of the excitation amplitudes that predicted the observed trends. We think this phenomenon is very promising for nanobalance applications where multicriterion systems are searched. Moreover, they can offer the possibility of addressing both single-particle level to perform local measurements and large-scale measurements. This kind of bimetallic system, once totally characterized, could be a good candidate for specific mass sensing applications needing robustness and versatility.

\section{METHODS}

The elongated bipyramidal nanoparticles are synthesized using a seed mediated technique in water. ${ }^{29}$ Gold seed nano spheres are first prepared by adding $0.3 \mathrm{~mL}$ of a fresh $10 \mathrm{mM}$ $\mathrm{NaBH}_{4}$ solution to $20 \mathrm{~mL}$ of a $0.125 \mathrm{mM} \mathrm{HAuCl}_{4}$ and $0.25 \mathrm{mM}$ sodium citrate solution under fast stirring. Seed solution is aged at least $5 \mathrm{~h}$ before use to allow total decomposition of $\mathrm{NaBH}_{4}$. Gold core nanoparticles are synthesized by injection of a small volume (between 15 and $80 \mu \mathrm{L}$ ) of seed solution to a growth solution prepared as follows. A $0.5 \mathrm{~mL}$ amount of $10 \mathrm{mM} \mathrm{HAuCl}_{4}$ is added to $10 \mathrm{~mL}$ of a $0.1 \mathrm{M} \mathrm{CTAB}$ solution followed by the addition of $0.2 \mathrm{~mL}$ of $1 \mathrm{M} \mathrm{HCl}$ and finalized by the addition of $0.08 \mathrm{~mL}$ of $0.1 \mathrm{ML}$ ascorbic acid. The growth is performed under gentle stirring at $35{ }^{\circ} \mathrm{C}$ for $2 \mathrm{~h}$. The cleaning process of the colloidal suspension is performed by centrifugation $(1500 \mathrm{~g}$ for 1 h $30 \mathrm{~min}$ ). The process is repeated until the CTAB concentration reaches $1 \mathrm{mM}$. Gold silver core shell nanoparticles were

prepared using a technique described elsewhere. ${ }^{30,31}$ An $80 \mathrm{mg}$ sample of PVP is added to $5 \mathrm{~mL}$ of a cleaned bipyramid solution and gently agitated for $20 \mathrm{~min}$. A volume of $1 \mathrm{mM} \mathrm{AgNO}_{3}$ solution (between 0.3 and $1 \mathrm{~mL}$ ) is then added, followed by the addition of $0.1 \mathrm{~mL}$ of $0.1 \mathrm{ML}$ ascorbic acid. The silver ion reduction is then initiated by reducing slowly the $\mathrm{pH}$ to 8 with dropwise addition of a $0.1 \mathrm{M} \mathrm{NaOH}$ solution. The reaction has to be very slow $(2 \mathrm{~h})$. It was previously observed that silver preferentially deposits onto lateral facets rather than the tips. ${ }^{31}$ Extinction spectroscopy is performed using a UV3600 Shimadzu in quartz cells. TEM micro scopy is performed using a TECNAI operating at $120 \mathrm{kV}$.

Time resolved experiments are performed using a femtose cond laser operating at a wavelength adjustable from 700 to $1000 \mathrm{~nm}$ delivering $150 \mathrm{fs}$ pulses at $80 \mathrm{MHz}$ repetition rate (Coherent Chameleon Ultra II). The output pulse train is sepa rated into two beams to create pump (100 $\mathrm{mW})$ and probe beams $(10 \mathrm{~mW})$, linearly cross polarized and focused in a $1 \mathrm{~mm}$ thick Hellma cell with 15 and $10 \mathrm{~cm}$ fused silica lenses, 
respectively. The pump induced transmission $\Delta T / T$ changes are detected using a standard pump probe setup with mechanical chopping of the pump beam at $2 \mathrm{kHz}$ and lock in differential detection (Stanford SR830) of the transmitted probe beam.

Conflict of Interest: The authors declare no competing financial interest.

Acknowledgment. The authors thank funding support from the French Conseil Régional d'Aquitaine (Project Nano Trans 20111101010), the Agence Nationale de la Recherche (Contract ANR BLAN SIMI10 LS 10061715 01) and CNRS. The authors thank the Nano Spectro Imagery facility (NSI) at LOMA.

Supporting Information Available: This document includes details on the methods used to determine the quality factor and information and data obtained with finite element analysis computations. This material is available free of charge via the Internet at http://pubs.acs.org.

\section{REFERENCES AND NOTES}

1. Krauss, T. D.; Wise, F. W. Coherent Acoustic Phonons in a Semiconductor Quantum Dot. Phys. Rev. Lett. 1997, 79, 51025105.

2. Bragas, A. V.; Aku Leh, C.; Merlin, R. Raman and Ultrafast Optical Spectroscopy of Acoustic Phonons in $\mathrm{CdTe} \mathrm{C}_{0.68} \mathrm{Se}_{0.32}$ Quantum Dots. Phys. Rev. B 2006, 73, 125305125310.

3. Gambetta, A.; Manzoni, C.; Menna, E.; Meneghetti, M.; Cerullo, G.; Lanzani, G.; Tretiak, S.; Piryatinski, A.; Saxena, A.; Martin, R. L. Real Time Observation of Nonlinear Co herent Phonon Dynamics in Single Walled Carbon Nano tubes. Nat. Phys. 2006, 2, 515520.

4. Major, T. A.; Crut, A.; Gao, B.; Lo, S. S.; Del Fatti, N.; Vallée, F.; Hartland, G. V. Damping of the Acoustic Vibrations of a Suspended Gold Nanowire in Air and Water Environments. Phys. Chem. Chem. Phys. 2013, 15, 41694176.

5. Del Fatti, N.; Voisin, C.; Christofilos, D.; Vallée, F.; Flytzanis, C. Acoustic Vibration of Metal Films and Nanoparticles. J. Phys. Chem. A 2000, 104, 43214326.

6. Portales, H.; Saviot, L.; Duval, E.; Fujii, M.; Hayashi, S.; Del Fatti, N.; Vallée, F. Resonant Raman Scattering by Breath ing Modes of Metal Nanoparticles. J. Chem. Phys. 2001, $115,34443447$.

7. Taubert, R.; Hudert, F.; Bartels, A.; Merkt, F.; Habenicht, A.; Leiderer, P.; Dekorsy, T. Coherent Acoustic Oscillations of Nanoscale Au Triangles and Pyramids: Influence of Size and Substrate. New J. Phys. 2007, 9, 376384.

8. Burgin, J.; Langot, P.; Arbouet, A.; Margueritat, J.; Mlayah, A.; Vallée, F.; Gonzalo, J.; Afonso, C. N.; Rossell, M.; et al. Acoustic Vibration Modes and Electron Lattice Coupling in Self Assembled Silver Nanocolumns. Nano Lett. 2008, 8, 12961302.

9. Pelton, M.; Sader, J.; Burgin, J.; Liu, M.; Guyot Sionnest, P.; Gosztola, D. Damping of Acoustic Vibrations in Gold Nanoparticles. Nat. Nanotechnol. 2009, 4, 492495.

10. Huang, W.; Qian, W.; El Sayed, M. A. Coherent Vibrational Oscillation in Gold Prismatic Monolayer Periodic Nanopar ticle Arrays. Nano Lett. 2004, 4, 17411747.

11. Hu, M.; Hillyard, P.; Hartland, G. V.; Kosel, T.; Perez Juste, J.; Mulvaney, P. Determination of the Elastic Constants of Gold Nanorods Produced by Seed Mediated Growth. Nano Lett. 2004, 4, 24932497.

12. Zijlstra, P.; Tchebotareva, A. L.; Chon, J. W. M.; Gu, M.; Orrit, M. Acoustic Oscillations and Elastic Moduli of Single Gold Nanorods. Nano Lett. 2008, 8, 34933497.

13. Ruijgrok, P. V.; Zijlstra, P.; Tchebotareva, A. L.; Orrit, M. Damping of Acoustic Vibrations of Single Gold Nanopar ticles Optically Trapped in Water. Nano Lett. 2012, 12, 10631069.

14. Marty, R.; Arbouet, A.; Girard, C.; Mlayah, A.; Paillard, V.; Lin, V. K.; Teo, S. L.; Tripathy, S. Damping of the Acoustic Vibrations of Individual Gold Nanoparticles. Nano Lett. 2011, 11, 33013306.

15. Burgin, J.; Langot, P.; Del Fatti, N.; Vallée, F.; Huang, W.; El Sayed, M. Time Resolved Investigation of the Acoustic
Vibration of a Single Gold Nanoprism Pair. J. Phys. Chem. C 2008, 112, 1123111235

16. Bonacina, L.; Callegari, A.; Bonati, C.; Van Mourik, F.; Chergui, M. Time Resolved Photodynamics of Triangular Shaped Silver Nanoplates. Nano Lett. 2006, 6, 710.

17. Jais, P. M.; Murray, D. B.; Merlin, R.; Bragas, A. V. Metal Nano particle Ensembles: Tunable Laser Pulses Distinguish Mono mer from Dimer Vibrations. Nano Lett. 2011, 11, 36853689.

18. Varnavski, O.; Ramakrishna, G.; Kim, J.; Lee, D.; Goodson, T. Optically Excited Acoustic Vibrations in Quantum Sized Monolayer Protected Gold Clusters. ACS Nano 2010, 4, 34063412.

19. Stalevaand, H.; Hartland, G. V. Vibrational Dynamics of Silver Nanocubes and Nanowires Studied by Single Parti cle Transient Absorption Spectroscopy. Adv. Funct. Mater. 2008, 18, 38093817.

20. Mongin, D.; Juvé, V.; Maioli, P.; Crut, A.; Del Fatti, N.; Vallée, F.; Sánchez Iglesias, A.; Pastoriza Santos, I.; Liz Marzán, L. M. Acoustic Vibrations of Metal Dielectric Core Shell Nanoparticles. Nano Lett. 2011, 11, 30163021.

21. Cardinal, M. F.; Mongin, D.; Crut, A.; Maioli, P.; Rodríguez González, B.; Pérez Juste, J.; Liz Marzán, L. M.; Del Fatti, N.; Vallée, F. Acoustic Vibrations in Bimetallic Au@Pd Core Shell Nanorods. J. Phys. Chem. Lett. 2012, 3, 613619.

22. Jones, M. R.; Osberg, K. D.; Macfarlane, R. J.; Langille, M. R.; Mirkin, C. A. Templated Techniques for the Synthesis and Assembly of Plasmonic Nanostructures. Chem. Rev. 2011, 111, 37363827.

23. Tao, A.; Habas, S.; Yang, P. Shape Control of Colloidal Metal Nanocrystals. Small 2008, 4, 310325.

24. Chaste, J.; Eichler, A.; Moser, J.; Ceballos, G.; Rurali, R.; Bachtold, A. A Nanomechanical Mass Sensor with Yocto gram Resolution. Nat. Nanotechnol. 2012, 7, 301304.

25. Guffey, M. J.; Miller, R. L.; Gray, S. K.; Scherer, N. F. Plasmon Driven Selective Deposition of Au Bipyramidal Nanoparti cles. Nano Lett. 2011, 11, 40584066.

26. Lombardi, A.; Loumaigne, M.; Crut, A.; Maioli, P.; Del Fatti, N.; Vallée, F.; Spuch Calvar, M.; Burgin, J.; Majimel, J.; Tréguer Delapierre, M. Surface Plasmon Resonance Prop erties of Single Elongated Nanoobjects: Gold Nanobipyr amids and Nanorods. Langmuir 2012, 28, 90279033.

27. Burgin, J.; Liu, M.; Guyot Sionnest, P. Dielectric Sensing with Deposited Gold Bipyramids. J. Phys. Chem. C 2008, 112, 1927919282.

28. Liu, M.; Guyot Sionnest, P.; Lee, T. W.; Gray, S. K. Optical Properties of Rodlike and Bipyramidal Gold Nanoparticles from Three Dimensional Computations. Phys. Rev. B 2007, 76, 235428235438.

29. Liu, M.; Guyot Sionnest, P. Mechanism of Silver(I) Assisted Growth of Gold Nanorods and Bipyramids. J. Phys. Chem. B 2005, 109, 2219222200.

30. Liu, M.; Guyot Sionnest, P. Preparation and Optical Proper ties of Silver Chalcogenide Coated Gold Nanorods. J. Mater. Chem. 2006, 16, 39423945.

31. Burgin, J.; Florea, I.; Majimel, J.; Dobri, A.; Ersen, O.; Tréguer Delapierre, M. 3D Morphology of Au and Au@Ag Nanobi pyramids. Nanoscale 2012, 4, 12991303.

32. Arbouet, A.; Voisin, C.; Christofilos, D.; Langot, P.; Del Fatti, N.; Vallée, F.; Lermé, J.; Celep, G.; Cottancin, E.; et al. Electron Phonon Scattering in Metal Clusters. Phys. Rev. Lett. 2003, 90, 177401177405

33. Juvé, V.; Scardamaglia, M.; Maioli, P.; Crut, A.; Merabia, S.; Joly, L.; Del Fatti, N.; Vallée, F. Cooling Dynamics and Thermal Interface Resistance of Glass Embedded Metal Nanoparticles. Phys. Rev. B 2009, 80, 195406195412.

34. Hu, M.; Wang, X.; Hartland, G. V.; Mulvaney, P.; Perez Juste, J.; Sader, J. E. Vibrational Response of Nanorods to Ultrafast Laser Induced Heating: Theoretical and Experimental Analysis. J. Am. Chem. Soc. 2003, 125, 1492514933.

35. Hartland, G. V. Coherent Vibrational Motion in Metal Parti cles: Determination of the Vibrational Amplitude and Ex citation Mechanism. J. Chem. Phys. 2002, 116, 80488055.

36. Crut, A.; Juvé, V.; Mongin, D.; Maioli, P.; Del Fatti, N.; Vallée, F. Vibrations of Spherical Core Shell Nanoparticles. Phys. Rev. B 2011, 83, 205430209439. 
37. Navarro, J. R. G.; Manchon, D.; Lerouge, F.; Cottancin, E.; Lermé, J.; Bonnet, C.; Chaput, F.; Mosset, A.; Pellarin, M.; Parola, S. Synthesis, Electron Tomography and Single Particle Optical Response of Twisted Gold Nano Bipyra mids. Nanotechnology 2012, 23, 145707145715.

38. Arbouet, A.; Del Fatti, N.; Vallée, F. Optical Control of the Coherent Acoustic Vibration of Metal Nanoparticles. J. Chem. Phys. 2006, 124, 144701144704.

39. Burg, T. P.; Godin, M.; Knudsen, S. M.; Shen, W.; Carlson, G.; Foster, J. S.; Babcock, K.; Manalis, S. R. Weighing of Biomo lecules, Single Cells and Single Nanoparticles in Fluid. Nature 2007, 446, 10661069.

40. Zijlstra, P.; Paulo, P. M. R.; Orrit, M. Optical Detection of Single Non Absorbing Molecules Using the Surface Plasmon Resonance of a Gold Nanorod. Nat. Nanotechnol. 2012, 7, 379382.

41. Ament, I.; Prasad, J.; Henkel, A.; Schmachtel, S.; Sönnichsen, C. Single Unlabeled Protein Detection on Individual Plasmonic Nanoparticles. Nano Lett. 2012, 12, 10921095.

42. Chakraborty, D.; van Leeuwen, E.; Pelton, M.; Sader, J. E. Vibration of Nanoparticles in Viscous Fluids. J. Phys. Chem. C 2013, 117, 85368544. 